\title{
Study on Fluorescence Characteristic of a Complex Probe of CdSe Quantum Dots Coupling with Thiazole Orange
}

\author{
X. Fei*, G. Jia, J. WAng And Y. Gu \\ Tianjin Institute of Urban Construction, Tianjin 300384, China \\ (Received December 22, 2009; in final form April 2, 2010)
}

\begin{abstract}
CdSe quantum dots were synthesized using thioglycolic acid as stabilizer in aqueous solution under $\mathrm{N}_{2}$. The UV-vis spectrometry and fluorescence spectra indicate that the bimodal quantum dots were formed and the optical band gaps are about $650 \mathrm{~nm}$ and $750 \mathrm{~nm}$, respectively. The quantum dots coated with TO were prepared in room temperature, and the fluorescence characteristic was studied. The result showed that the peak shift of quantum dots fluorescence spectra can mainly be due to the change of the capping layer, resulting in the confinement energy change. This is vital for the investigating on of the forming process and mechanisms of the combination of thiazole orange dye and quantum dots.
\end{abstract}

PACS numbers: 73.63.Kv, 78.55.-m, 78.67.-n

\section{Introduction}

The research of Fluorescence characteristic of organic dye conjugated by colloidal semiconductor nanocrystals quantum dots (QDs) is very important since it offers a powerful technique for probing the distance change between donor and acceptor [1]. QDs have attracted much attention due to their unique advantages such as: narrow photoemission, strong fluorescence, broad photoexcitation, and high resistance to photobleaching. The QDs can be commonly prepared in organic solvents at high temperature and then functionally modified. Although highly stable water-soluble QDs can be prepared in this way, the size of QDs is relatively large. Recently, the water-dispersed QDs directly synthesized in the aqueous phase are of great interest and have been widely investigated $[2,3]$. In order to improve the photoluminescence quantum yield and stability, $\mathrm{CdTe} / \mathrm{CdS}$ and $\mathrm{CdTe} / \mathrm{CdS} /$ ZnS core-shell composite QDs have been successfully prepared in an aqueous phase assisted by microwave irradiation [2], which exhibit excellent photostability and favorable biocompatibility. In addition, the broad absorption and narrow emission spectra of the QDs make them excellent donors in fluorescence resonance energy transfer (FRET)-based sensors, because these fluorescence characteristics allow the selection of a wide range of excitation wavelengths to minimize the background due to direct excitation of the acceptor $[4,5]$. Hering et al. reported that fluorescent proteins from the green fluorescent protein (GFP) family interact strongly with $\mathrm{CdSe} / \mathrm{ZnS}$ quantum

\footnotetext{
* corresponding author; e-mail: xueningfei888@126.com
}

dots. It is not clear yet whether FRET is the reason behind the quenching when the degree of quenching was elevated [6]. Especially, to Thiazole Orange (TO) as an important fluorescent dye, that a detailed investigation is necessary to understand the mechanism of fluorescence resonance energy transfer between QDs and linked TO acceptors.

In this paper, CdSe core/shell composite QDs were synthesized in aqueous solution with thioglycolic acid as stabilizer under $\mathrm{N}_{2}$. X-ray diffraction was used to characterize the structure of QDs. The complex probe was prepared by the synthesized QDs coupling with TO at room temperature and the fluorescence characteristics of the complex probe was analyzed preliminarily.

\section{Experimental details}

All chemicals were used without further purification. Tellurium power $(99.9 \%), \mathrm{CdCl}_{2}(99.9 \%)$, and thioglycolic acid (98\%) were purchased from Tianjin Chemical Reagents Company. Distilled water was used in the experiment. Briefly, the water-soluble CdSe quantum dots were synthesized by using thioglycolic acid as stabilizer in aqueous solution under $\mathrm{N}_{2}$ because QDs have high affinity for thiols [7]. The quinoline derivatives with amine group reacted with the QDs containing carboxylic acid functional group. Then benzothiazole derivative was added to form the title compound (QD-TO), which is composed of QDs coupling with TO with the help of condensing agent.

XRD patterns were obtained using Rigaku K/max-rA diffractometer with $\mathrm{Cu}$ Ka radiation for $2 \theta$ values over $20-80^{\circ}$. All fluorescence spectra were recorded on a Cary 
Eclipse Fluorescence Spectrophotometer at fixed excitation wavelength of $338 \mathrm{~nm}$. The emission spectra were recorded between 500 and $800 \mathrm{~nm}$ at a scan rate of $2 \mathrm{~nm} / \mathrm{s}$ and slit width was $5 \mathrm{~nm}$. An excitation and emission bandwidth of $5 \mathrm{~nm}$ was used. The Fourier transform infrared (FTIR) spectrum was measured on a Thermo Nicolet 380 spectrometer.

\section{Results and discussion}

Figure 1 shows the XRD spectra of CdSe nanocrystals. The narrow lines come from the Si standard for the CdSe sample. The powder diffraction of the samples exhibits three peaks that can be assigned to (111), (220), and (311) reflections of the zinc blende structure. The average crystallite size calculated by Scherer formula is about $12.9 \mathrm{~nm}$

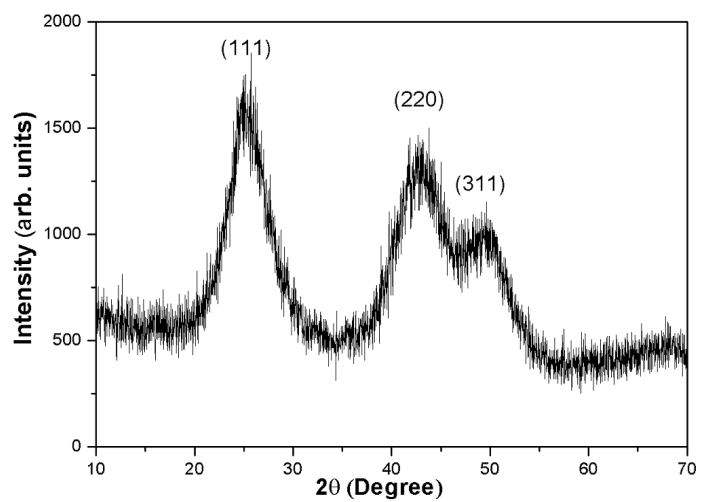

Fig. 1. XRD pattern of CdSe nanoparticles.

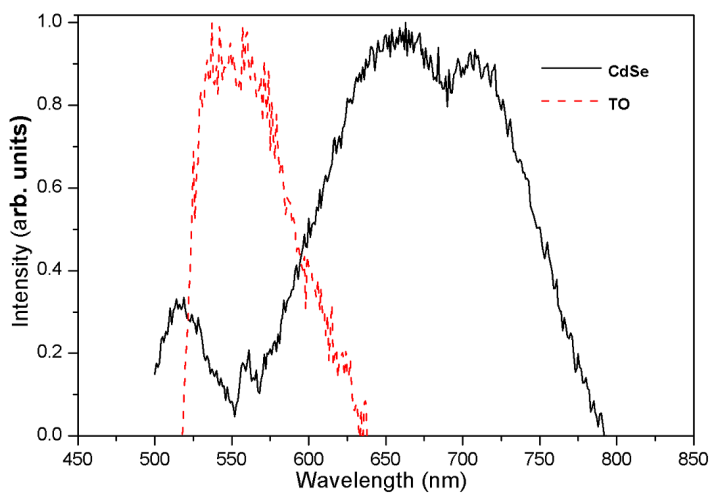

Fig. 2. The fluorescence spectra of the CdSe sample and TO.

Figure 2 displays emission spectra of the CdSe sample and TO excited at $328 \mathrm{~nm}$. The emission spectrum of CdSe QDs shows that the bimodal size distribution of QDs was formed. Obviously, the stabilizer plays a critical role in the forming of bimodal QDs. The ripening of QDs needed a procession, therefore, the bimodal size distribution QDs were formed, one part with optimum size, the other without ripening [8]. The emission spectrum of
QDs overlaps with the absorption spectrum of TO while still maintaining good spectral resolution of the donor and acceptor emission.

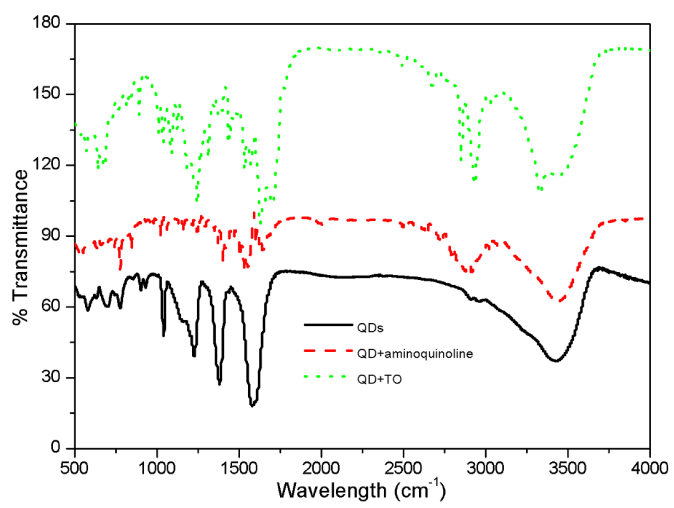

Fig. 3. FTIR spectra of CdSe QDs, QDs-quinoline and QDs-TO composites.

The samples of CdSe QDs with thioglycolic acid capping layer, CdSe QDs with quinoline bearing amine group capping layer, and CdSe QDs with TO capping layer were characterized by FTIR spectra shown in Fig. 3. The peak at $1590 \mathrm{~cm}^{-1}$ should be coincident with carbonyl stretching vibration, which shows that the CdSe QDs are bound with the carboxylic acid functional group of thioglycolic acid $[9,10]$. Compared with the QDs capped with thioglycolic acid, the - $\mathrm{CO}-\mathrm{NH}$ mode contains contributions from $\mathrm{C}-\mathrm{N}$ stretching vibration and $\mathrm{N}-\mathrm{H}$ bending vibration of the CdSe QDs with TO capping layer. When the benzothiazole derivative was added into the solution of the QDs with capping layer of quinoline bearing amine group, the title compound was formed.

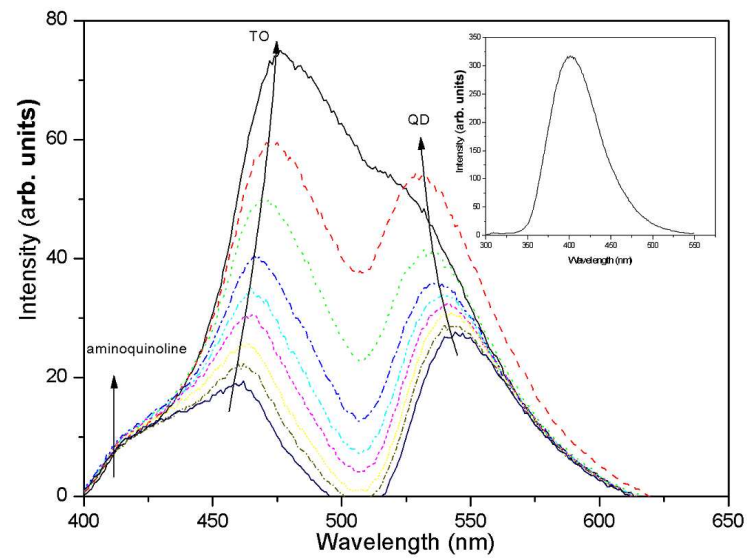

Fig. 4. Photoluminescence spectra of the TO donor and the corresponding QD acceptor as a function of increasing benzothiazole derivatives to $\mathrm{QD}$, the quantity of the benzothiazole was increased from down to up. The photoluminescence spectra obtained from the quinoline (inset). 
Thiazole Orange a benzothiazole ring covalently linked to a quinoline ring through a monomethine bridge, has been widely used as the embedded cyanine dye for labeling nucleic acids, allowing the detection of DNA and RNA in gels, flow cytometry, or microscopy [11]. Although the fluorescence of free TO is extremely low in aqueous solution, when TO binds to nucleic acids, the viscosity of the dye's local environment is markedly increased, resulting in a drastic increase in fluorescence [12]. Figure 4 shows the changes in the ensemble emission spectra of conjugated CdSe with the increase of benzothiazole derivative amount. The concentration of QDs was kept constant through the experimental process. The maximum emission wavelength of quinoline remained constant, while TO and QDs fluorescent signal increased with the benzothiazole being added, which can be explained by analyzing the mechanism of TO forming. TO increases with addition of benzothiazole because the quinoline reacted with benzothiazole to form TO with the help of condensing agent. Obviously, the fluorescence phenomenon caused by TO and QDs is owing to the domination of a fast radiative decay rate of the TO dye excitation relative to a slow FRET decay rate [1]. The increase of QDs fluorescence intensity is mainly due to its overlap with the fluorescence of TO. Additionally, a red shift of the TO fluorescence peak and a blue shift of the QDs were observed with the increasing concentration of benzothiazole. As far as TO is concerned, the peak shift results from the change of structure, which is due to the confinement energy's change by altering the cap layer from quinoline to TO.

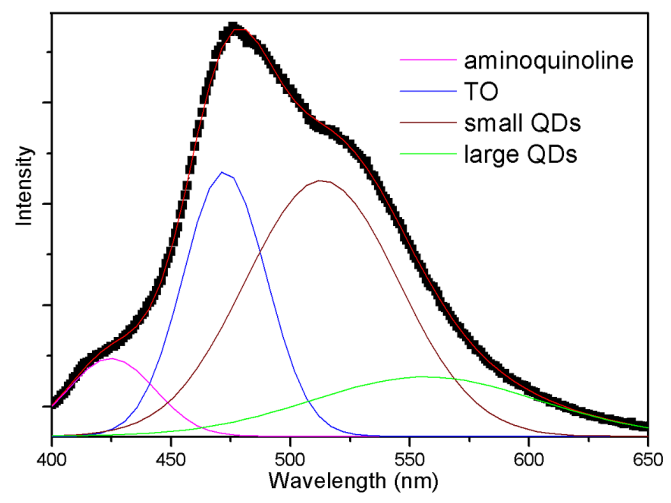

Fig. 5. Typical photoluminescence spectra from Fig. 4, is fitted to the sum of four Gaussian lineshapes.

Figure 5 shows the photoluminescence spectra of the system with different energy and intensity. The spectra is fitted to the sum of four Gaussian lineshapes, which can be ascribed as the emission of quinoline bearing amine group (about $420 \mathrm{~nm}$ ), TO (about $470 \mathrm{~nm}$ ), QDs (about $565 \mathrm{~nm}$ and $560 \mathrm{~nm}$ ), respectively. The higher energy emission (about $565 \mathrm{~nm}$ ) originated from smaller size and stronger density QDs, while lower energy emission (about $560 \mathrm{~nm}$ ) corresponded to larger size and weaker intensity QDs. It can also be found that the size distribution of smaller QDs family is narrower than that of larger QDs family.

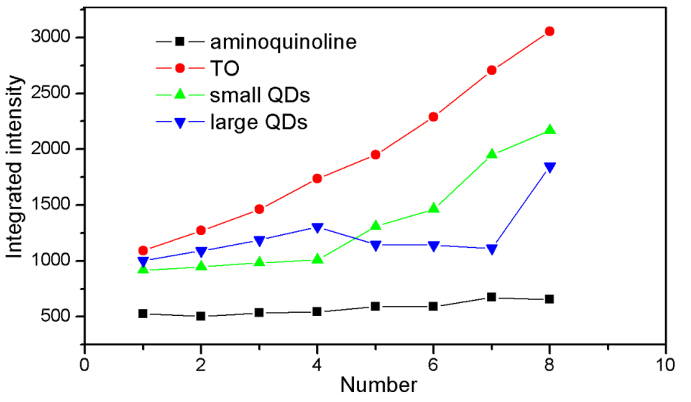

Fig. 6. The benzothiazole concentration dependence of integrated intensity for the different components.

In order to further analyze the fluorescence caused by TO organic dye and CdSe quantum dots, the benzothiazole concentration depending on integrated intensity was shown in Fig. 6. It should be noted that the concentration of benzothiazole can't be predicted accurately. A simple analysis of the change of integrated intensity with the increase of benzothiazole was carried out here. For quinoline, the integrated intensity had no obvious change due to little effect of benzothiazole, while the integrated intensity of the small QDs increase with the increase of the benzothiazole concentration.

\section{Conclusions}

In conclusion, CdSe QDs were synthesized in aqueous solution by using thioglycolic acid stabilizer under $\mathrm{N}_{2}$. It was verified that the QDs have the good crystalline structure through X-ray diffraction. The fluorescence spectra indicate that the bimodal QDs were formed. The changes in the ensemble emission spectra of conjugated CdSe with the increase of benzothiazole derivative amount show a red shift of the TO fluorescence peak and a blue shift of the QDs.

\section{Acknowledgments}

This work is financially supported by the National Natural Science Foundation of China (No. 20772090), Key Project of Science and Technology Committee of Tianjin (No. 08JCZDJC18200), the Natural Science Foundation of Tianjin (No. 09JCYBJC04100), Science and Technology Plan Projects of the Ministry of Construction of China (No. 2008-KT-11).

\section{References}

[1] A.R. Clapp, I.L. Medintz, B.R. Fisher, H. Mattoussi, J. Am. Chem. Soc. 127, 1242 (2005).

[2] Y. He, H.T. Lu, L.M. Sai, Y.Y. Su, M. Hu, C.H. Fan, W. Huang, L.H. Wang, Adv. Mater. 20, 3416 (2008).

[3] Z. Fang, L. Liu, L. Xu, X. Yin, X.H. Zhong, Nanotechnology 19, 235603 (2008). 
[4] D.J. Zhou, L.M. Ying, X. Hong, Langmuir 24, 1659 (2008).

[5] A.M. Dennis, G. Bao, Nano lett. 8, 1439 (2008).

[6] V.R. Hering, G. Gibson, R.I. Schumacher, A. Faljoni-Alario, M.J. Politi, Bioconjugate Chem. 18, 1705 (2007).

[7] D. Zhou, L.M. Ying, X. Hong, E.A. Hall, C. Abell, D. Klenerman, Langmuir 24, 1659 (2008).

[8] G.Z. Jia, J.H. Yao, Y.C. Shu, X.D. Xing, B. Pi, Appl. Surf. Sci. 255, 4452 (2009).
[9] W.W. Wright, M. Laberge, J.M. Vanderkooi, Biochemistry 36, 14724 (1997).

[10] Y. Lei, H.Y. Tang, C.J. Zhou, T.T. Zhang, M.F. Feng, B.S. Zou, J. Lumin. 128, 277 (2008).

[11] L.G. Lee, C.H. Chen, L.A. Chiu, Cytometry 7, 508 (1986).

[12] J. Nygren, N. Svanvik, M. Kubista, Biopolymers 46, 39 (1998). 